\title{
The Beta-Decay Induced by Neutrino Flux
}

\author{
Boris V. Vasiliev \\ Dubna, Russia \\ Email: bv.vasiliev@yandex.com
}

How to cite this paper: Vasiliev, B.V. (2020) The Beta-Decay Induced by Neutrino Flux. Journal of Modern Physics, 11, 608-615. https://doi.org/10.4236/jmp.2020.115040

Received: April 5, 2020

Accepted: May 5, 2020

Published: May 8, 2020

Copyright $\odot 2020$ by author(s) and Scientific Research Publishing Inc. This work is licensed under the Creative Commons Attribution International License (CC BY 4.0).

http://creativecommons.org/licenses/by/4.0/

\begin{abstract}
Previously, it has been repeatedly suggested that the radioactive decay of nuclei is not a random phenomenon and can occur under the influence of a neutrino flux. Our recent experiment [1] showed that the neutrino flux created by a nuclear reactor affects the decay of nuclei in an isolated source ${ }^{90} \mathrm{Sr} /{ }^{90} \mathrm{Y}$, whose beta-electrons have an average energy of the order of $1 \mathrm{MeV}$. This paper presents the results of searching for the effect of the neutrino flux generated by a pulsed nuclear reactor on the rate of decay of ${ }^{63} \mathrm{Ni}$ nuclei. These nuclei were chosen as the object of research due to the fact that they have a low energy of beta-electrons of the order of $50 \mathrm{keV}$. Measurements have shown that the same flux of reactor neutrinos has approximately an order of magnitude stronger effect on ${ }^{63} \mathrm{Ni}$ nuclei than on the previously studied nuclei ${ }^{90} \mathrm{Sr} /{ }^{90} \mathrm{Y}$.
\end{abstract}

\section{Keywords}

Nuclear Reactor, Neutrino Flux, Beta-Decay, Isotope ${ }^{63} \mathrm{Ni}$

\section{Introduction}

The discovery of radioactivity raised the question: is radioactive decay a random phenomenon?

The new physics of the early twentieth century gave the phenomenon of radioactive decay a quantum-mechanical explanation. N. Bohr and other creators of quantum mechanics explained it by the laws of tunnel phenomena and considered its absolute randomness to be proved.

The power of the quantum mechanics apparatus and the beauty of the new approach have swayed the physical community to the side of the anti-determinists. Currently, this view is dominant and radioactive decay is considered a purely random phenomenon.

But some other physicists from the very beginning of discussion did not agree with this approach. 
A. Einstein expressed his categorical rejection of the new point of view with his famous metaphor: “God doesn't play dice!”

From a deterministic point of view, radioactive decay must have been caused by some hitherto unknown external causes.

The neutrino flux is very well suited for the description of the external causes that can induce beta-decay.

The hypothesis that the impact of the neutrino flux may be the cause of beta-decay of radioactive nuclei has been repeatedly expressed earlier by various researchers [2] [3] [4].

It is important that the phenomenon of beta-decay of neutron is energetically advantageous, since the mass of neutron is greater than the total mass of proton and electron.

All main parameters of neutron, its mass, spin, magnetic moment, decay energy, can be calculated with satisfactory accuracy in the framework of the electromagnetic model [5] [6] [7]. This model predicts the characteristic properties of neutron excited states [6] that it should possess. In high-energy physics, particles with these properties are commonly called hyperons.

(Only the neutron lifetime is not computable).

Electromagnetic forces that, according to the electromagnetic model, bind proton and electron to form neutron do not carry a mechanism for degradation of the combined state.

In this model, there is no internal cause that could induce the lose stability of neutron and its decay.

Therefore, in order to cause the decay of neutrons or $\beta$-active nuclei, they must be affected from the outside by the neutrino flux.

In previous paper, it was shown that the flux of reactor neutrinos does have a small effect on the rate of beta-decay in the source ${ }^{90} \mathrm{Sr} /{ }^{90} \mathrm{Y}[1]$.

In this paper, it is shown that the same flux of reactor neutrinos has approximately an order of magnitude stronger effect on the decay of ${ }^{63} \mathrm{Ni}$ nuclei, characterized by low energy beta-electrons.

\subsection{The Neutron Lifetime}

Multiple studies of the neutron lifetime have shown one feature. Neutrons in the last century at very accurate measurements made on relatively small reactors lived noticeably longer than neutrons lived near the powerful Grenoble reactor at the beginning of the XXI century.

The difference in life time of these neutrons in these changes significantly went beyond the limits of experimental errors [8]. This result can be explained by the fact that the powerful neutrino flux of the Grenoble reactor causes accelerated neutron decay.

\subsection{The Effect of Solar Neutrinos on $\beta$-Decay of Nuclei}

A clear result was obtained by E. Fishbach and J. Jenkins [3]. 
They showed that according to the data of experiments conducted in various laboratories around the world, the rate of nuclei decay depends on the distance between the Sun and the Earth. The authors explained this effect by the influence of the solar neutrino flux.

Accordingly to measurement data [9], the sunny neutrino flux is equal approximately

$$
\Phi_{\odot} \approx 6 \times 10^{10} v / \mathrm{cm}^{2} \cdot \mathrm{s}
$$

To explain the measured modulation depth, it can be assumed that the solar neutrino flux is approximately only $1 / 30$ of the total neutrino flux that affects the decay of nuclei in our laboratories, and this total neutrino flux from all cosmic sources is equal to

$$
\Phi_{\text {cosm }} \approx 30 \cdot \Phi_{\odot} \approx 2 \times 10^{12} \mathrm{v} / \mathrm{cm}^{2} \cdot \mathrm{s}
$$

\section{The Effect of Reactor Neutrinos on $\beta$-Decay}

\section{Experiments on a Stationary Reactor}

In order to detect the effect of neutrino flux on the beta-decay phenomenon, a group of researchers led by E. Fishbach conducted experiments on the High Flux Isotope Reactor of Oak Ridge National Laboratory [10].

This reactor had a high power

$$
W_{\text {HFIR }} \approx 85 \mathrm{MW} \approx 1.4 \times 10^{21} \mathrm{MeV} / \mathrm{s} .
$$

Given that the fission of a single nucleus of reactor fuel releases approximately $200 \mathrm{MeV}$ of energy [11], this reactor produced approximately $10^{19}$ of nuclear fission per second.

If we assume that one act of fission produces 6 neutrino [11], it turns out that at a conditional distance of 10 meters from the core, this reactor created a neutrino flux

$$
\Phi_{\text {HFIR }} \approx 3 \times 10^{12} v / \mathrm{cm}^{2} \cdot \mathrm{sec}
$$

Thus, the neutrino flux generated by this reactor was approximately equal to the flux of cosmic neutrino (Eq. (2)).

Therefore, according to the authors, switching the reactor on or off should have greatly changed the rate of decay in the beta sources under study.

However, despite a very serious approach to the processing of experimental results, the authors did not find any effect of such a powerful reactor on the beta-decay of a number of studied nuclei [10].

How this discrepancy in research results ([3] and [10]) can be explained? The possible reason for this is due to the difference in the spectra of cosmic and reactor neutrinos.

Most reactor neutrinos generated by fission fragments have an energy of the order of $1 \mathrm{MeV}$ or less. At the same time among the cosmic neutrinos, there are particles with very high energy.

In this regard, it can be assumed that the average flow of reactor neutrinos 
may have a smaller effect on beta-decay than the same-valued flow of cosmic neutrinos.

In this regard, in order to notice the effects of reactor neutrinos on beta-decay, it is necessary to take a different approach to this experiment.

\section{Experiments on a Pulsed Reactor}

\subsection{The Pulse Reactor Efficiency}

For this experiment, we used the IBR-2 reactor (Dubna) as a neutrino source. It has an average power of $1.6 \mathrm{MW}$ and operates in pulse mode. The interval between the flashes was about $200 \mathrm{~ms}$.

The duration of the flash itself is approximately $0.3 \mathrm{~ms}$.

The estimate shows that the neutrino flux generated by this reactor during the flash of its intensity is approximately equal to the flow of a powerful stationary reactor or the flow of cosmic neutrinos.

Using a pulse reactor makes it possible to accumulate information synchronously with the flash. This is an important advantage because over a long time of measurement, it is possible to sum up the data obtained in the on-off mode of the reactor.

This method of data accumulation is equivalent to the increasing of the reactor power by several orders of magnitude.

\subsection{The Reactor Neutrinos Generation}

During the fission reaction of nuclear fuel, $\beta$-active nuclei are born in the form of fission fragments. Their decay leads to the generation of neutrinos.

However, almost all fission fragments of nuclear fuel have a half-life longer than the time period separating the flares of our reactor. Therefore, in our case, they can be considered long-lived. Their decays are not "tied" to a specific flash of the reactor and can only give a slight increase in the background in the accumulation mode.

There are very few short-lived isotopes with a half-life of $T_{1 / 2}<200 \mathrm{~ms}$ in nature. One of these isotopes, ${ }^{12} \mathrm{~B}$, has a half-life of about $20 \mathrm{~ms}$ [12].

The probability of its formation as a plutonium fission fragment is unknown and should probably be very small.

But it is a great success that the neutrinos formed during its decay have a high boundary energy-up to $13.3 \mathrm{MeV}$ [12].

It is likely that the beta-decay of ${ }^{12} \mathrm{~B}$ creates those neutrinos whose effect on the beta-decay at the source of ${ }^{90} \mathrm{Sr} /{ }^{90} \mathrm{Y}$ was registered earlier (Figure 1) [1].

The measurement time is 3 days.

\section{Experimental Setup and Measurement Results}

A new series of experiments was carried out on the same installation that had previously been used for measurements effects of the neutrino flux on the decay of nuclei ${ }^{90} \mathrm{Sr} /{ }^{90} \mathrm{Y}[1]$. 


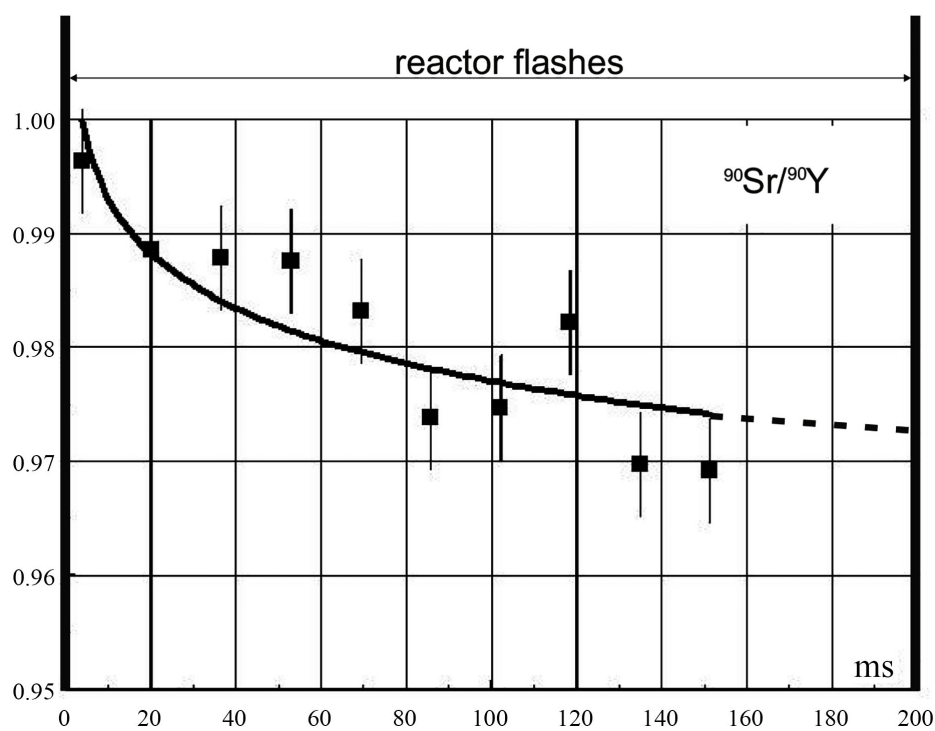

Figure 1. The result of the accumulation of registered beta-electrons emitted by the ${ }^{90} \mathrm{Sr} /{ }^{90} \mathrm{Y}$ source in the time interval between reactor flashes.

In new experiment, the rate of $\beta$-decays in the source ${ }^{63} \mathrm{Ni}$ was measured.

Beta-electrons emitted by ${ }^{63} \mathrm{Ni}$ nuclei were registered using a scintillation detector equipped with a photomultiplier (Figure 2).

Using electronics, the photomultiplier pulses were amplified, discriminated in amplitude, and then registered.

In order to protect the sensitive part of the equipment from the penetration of neutrons and $\gamma$-quanta, it was placed in a small house made of lead bricks and borinated polyethylene.

The measuring system was located at a distance of about 20 meters from the core of the pulse reactor.

In the absence of the $\beta$-source, the measuring system gave false positives no more than once every few minutes when the reactor was running.

When the reactor was switched off, beta decays in the source occurred statistically evenly.

The results of measurements of the effect of reactor neutrinos on the decay rate of the ${ }^{63} \mathrm{Ni}$ isotope are shown in Figure 3.

This figure shows a peak of reactor activity, generated approximately $3 \mathrm{~ms}$ after the start of data logging. The figure shows that before the reactor flash, the beta-electron count occurs at a certain low level, determined by the established amplitude discrimination.

Without discrimination, the beta-electron count, determined by the activity of the source, is about 6 orders of magnitude higher.

The reactor pulse generates a spike in the rate of decay in the source, followed by the linear decline (on a logarithmic scale, i.e. exponential on a linear scale) of the account. This exponent corresponds to a half life of approximately $20 \mathrm{~ms}$, which corresponds to the reference data for the isotope ${ }^{12} \mathrm{~B}[12]$. 


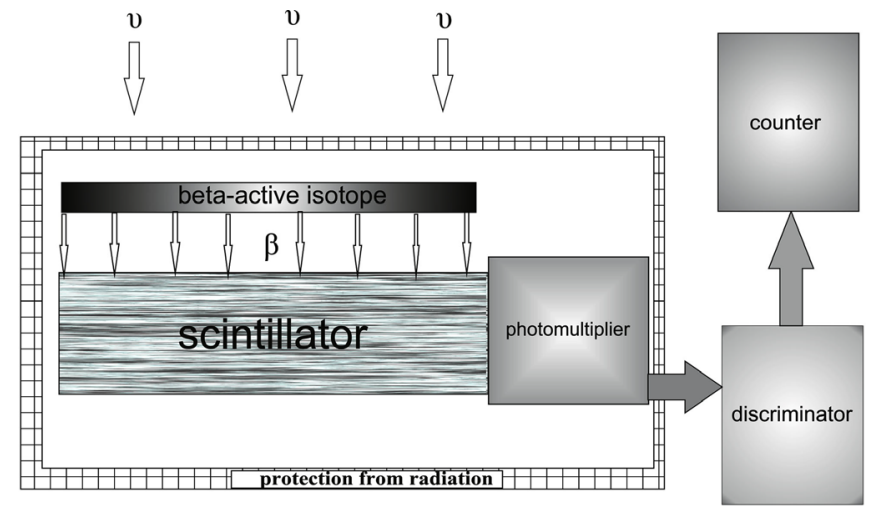

Figure 2. Diagram of the measuring unit.

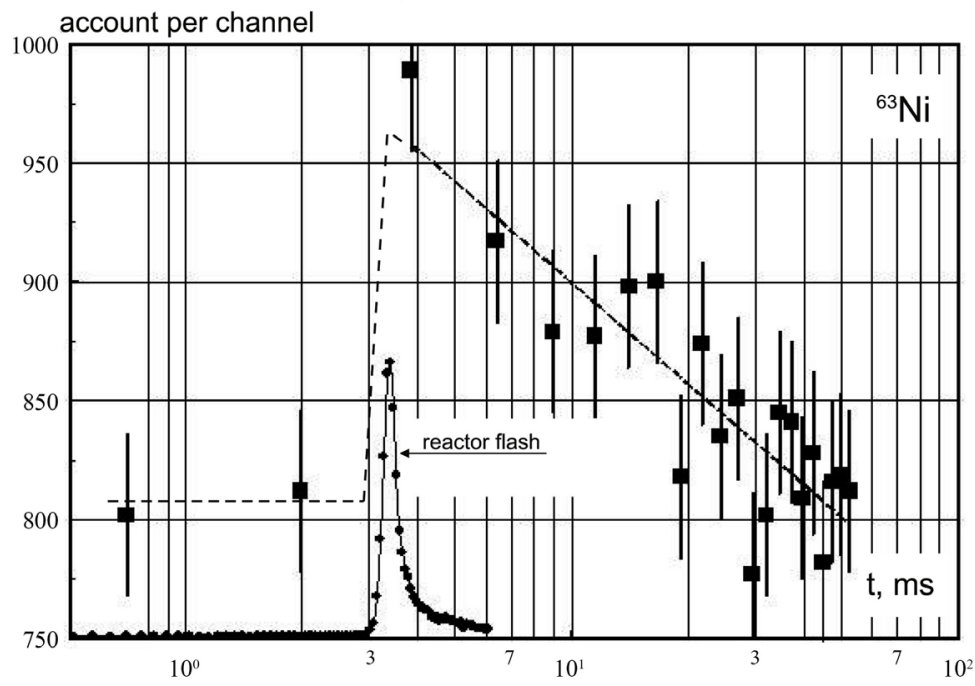

Figure 3. The result of the accumulating registration of beta-electrons emitted by ${ }^{63} \mathrm{Ni}$. Measurement time is 1 day. The level of amplitude discrimination close to the boundary energy was chosen expermentally. On abscissa: time in $\mathrm{ms}$ in the logarithmic scale.

As known the boundary energy of beta-electrons of the ${ }^{63} \mathrm{Ni}$ isotope is only $0.067 \mathrm{MeV}$, while the boundary energy of beta-electrons of ${ }^{90} \mathrm{Sr} /{ }^{90} \mathrm{Y}$ isotope is approximately $2.3 \mathrm{MeV}$.

When selecting the ${ }^{63} \mathrm{Ni}$ isotope as an object for studying we assumed that the effect of the neutrino flux on an isotope with a lower boundary energy would be stronger. It turned out to be correct.

There seems to be some approximately inverse relationship between the number of beta-electrons generated by reactor neutrinos and their energy.

The physics of this dependence seems to be related to the fact that when neutrino collides with nucleus, which leads to the decay of this nucleus, some part of the neutrino's energy is ultimately transferred to the beta-electron.

And the probability of such energy transfer is greater for small portions of it.

The average energy of a reactor neutrino can be equal to $1 \mathrm{MeV}$ or more.

However, since only a small portion of this energy reaches beta-electron, in 
order to register this effect, it is advisable to study it on isotopes with low energy of beta-electrons.

As seen in Figure 3 the decay total account of ${ }^{63} \mathrm{Ni}$ increases under the action of reactor neutrinos by about a quarter per 1 day. The same flux of reactor neutrinos affects the rate of decay of ${ }^{90} \mathrm{Sr} /{ }^{90} \mathrm{Y}$ isotopes about ten times weaker (Figure 1).

\section{Conclusions}

In response to A. Einstein's metaphorical statement, anti-determinists developed quantum mechanical theory of alpha-decay.

The power of the quantum mechanical apparatus and the novelty of this approach have tilted public opinion to the side of anti-determinists.

As a result currently, in the physical community, most believe that radioactive decay is a truly random process.

However, this issue should not be decided by voting.

The way to solve such problems was determined more than 400 years ago by the outstanding scientist of the middle ages W. Gilbert (1544-1603), who formulated the basic principle of natural sciences:

"Theoretical constructions that claim to be scientific must be tested and confirmed experimentally."

The dispute between determinists and anti-determinists about the nature of nuclear decay can be resolved experimentally only.

It seems that the discovered effect of reactor neutrinos on the rate of beta-decay suggests that the nature of this phenomenon was correctly understood by A. Einstein.

Now it seems that the study of the possible influence of reactor neutrinos on the phenomenon of alpha-decay is of great interest.

The author is sincerely grateful to A.V. Strelkov and V. V. Zhuravlev for preparing the high-purity ${ }^{63} \mathrm{Ni}$ beta-source and realization of measurements at the reactor.

\section{Conflicts of Interest}

The author declares no conflicts of interest regarding the publication of this paper.

\section{References}

[1] Vasiliev, B.V. (2020) Journal of Modern Physics, 11, 91-96. https://doi.org/10.4236/jmp.2020.111005 https://www.scirp.org/Journal/PaperInformation.aspx?PaperID=97775

[2] Falkenberg, E.D. (2001) Apeiron, 8, 32-45. https://pdfs.semanticscholar.org/3a21/346203f836847e60016770d931b324a46be9.pd $\underline{\mathrm{f}}$

[3] Jenkins, J.H., et al. (2008) Evidence for Correlations between Nuclear Decay Rates and Earth-Sun Distance. arXiv:0808.3283v1 [astro-ph] 
https://doi.org/10.1016/j.astropartphys.2009.05.004

https://arxiv.org/abs/0808.3283

[4] Vasiliev, B.V. (2017) Journal of Modern Physics, 8, 338-348.

https://doi.org/10.4236/jmp.2017.83023

http://www.scirp.org//ournal/PaperInformation.aspx?PaperID=74443

[5] Vasiliev, B.V. (2015) Journal of Modern Physics, 6, 648-659.

https://doi.org/10.4236/jmp.2015.65071

http://www.scirp.org/Journal/PaperInformation.aspx?PaperID=55921

[6] Vasiliev, B.V. (2019) Journal of Modern Physics, 10, 1487-1497.

https://www.scirp.org/journal/paperinformation.aspx?paperid=96226

https://doi.org/10.4236/jmp.2019.1013098

[7] Vasiliev, B.V. (2017) Journal of Nuclear Science, 1, 2.

http://scifed.com/fulltext/the-electromagnetic-model-of-neutron-and-nature-of-nu clear-forces $/ 21910$

https://doi.org/10.23959/sfjns-1000013

[8] Serebrov, A.P. (2005) Physics-Uspekhi, 48, 867. http://ufn.ru/ru/articles/2005/9/a/ https://doi.org/10.1070/PU2005v048n09ABEH003536

[9] Ahmed, S.N., Anthony, A.E., Beier, E.W., et al. (2004) SNO Collaboration. arXiv:nuclyex 0309004.

[10] Barnes, V.E., et al. (2019) Applied Radiation and Isotopes, 149, 182-199. https://doi.org/10.1016/j.apradiso.2019.01.027

[11] Hayes, A.C. and Vogel, P. (2016) Reactor Neutrino Spectra. arXiv 1605.02047v1 https://arxiv.org/pdf/1605.02047.pdf https://doi.org/10.1146/annurev-nucl-102115-044826

[12] Kikoine, I.K., a.o. (1978) Physical Tables, Moscow, Atomizdat. (In Russian) 